\title{
CHAOTIC BEHAVIOR IN A SINGULARLY PERTURBED SYSTEM
}

\author{
IGNACIO BOSCH VIVANCOS ANTONMARÍA A. MINZONI
}

\begin{abstract}
The aim of this paper is to present a novel type of chaos observed in a four dimensional singularly perturbed system. The chaotic behavior is described both numerically and analytically. We show the existence of a new type of strange attractor reminiscent of a chaotic behavior for a fast-slow system. Related to this we demonstrate that there is an invariant attracting region overlapping the jump manifolds of the fast system. This canonical problem was identified to be a coupled problem of two Hopf bifurcations with the fast jump process. We derive a geometric model where we can rigorously show the existence of chaotic orbits.
\end{abstract}

\section{INTRODUCTION}

Most mathematical analysis of qualitative properties of singularly perturbed systems done so far deal with local studies of phenomena occurring in system equations of low dimensions, see Guckenheimer [5] for a nice survey on this topic. Our goal in this work is to extend the local analysis and show how interesting global features can appear in four dimensional singularly perturbed systems. In particular, one of the main characteristics of singular systems is the fact that they possess different time scales. As a consequence, two dynamically different manifolds appear: a slow manifold where the system varies slowly and a fast manifold generally of lower dimension. Bifurcations in the family of vector fields induce transitions of trajectories from the neighborhood of one family of attractors to another on the fast time scale. To our knowledge, little is known about systems presenting many transition orbits between these attractors. We do not pretend in this work to make a rigorous analysis of such transitions but rather to exhibit global properties and, in particular, the existence of high dimensional strange attractors.

In this paper we concentrate our efforts on both the analytical and numerical studies of the following singularly perturbed system:

$$
\left\{\begin{array}{c}
\dot{x}_{1}=x_{1}\left(\lambda+a x_{1}^{2}+b x_{2}^{2}+c x_{3}^{2}\right)+p_{1} x_{2} x_{3} u \\
\dot{x}_{2}=x_{2}\left(\lambda+a x_{2}^{2}+b x_{3}^{2}+c x_{1}^{2}\right)+p_{1} x_{1} x_{3} u \\
\dot{x}_{3}=x_{3}\left(\lambda+a x_{3}^{2}+b x_{1}^{2}+c x_{2}^{2}\right)+p_{1} x_{1} x_{2} u \\
\varepsilon \dot{u}=u\left(\mu-u^{2}+p_{2}\left(x_{1}^{2}+x_{2}^{2}+x_{3}^{2}\right)\right)+p_{3} x_{1} x_{2} x_{3},
\end{array}\right.
$$

$a, b, c, \lambda, \mu$ and $\varepsilon(1>>\varepsilon \geq 0)$ will be fixed while $p_{1}, p_{2}, p_{3}$ denote parameters. The first motivation for the study of such system equations comes from a model of planar convection undertaken by Busse and Heikes [2] in 1980. Their mathematical

Date: October 18, 2003.

Key words and phrases. Singular Perturbations, Hopf bifurcation, Chaos.

The essence of this paper was conceived whilst working in the FENOMEC project. 
model was intended to mimic the instability (Kuppers-Lortz instability) observed for convection rolls in a rotating layer leading to a three dimensional system of ODEs for the amplitudes $\left(x_{1}, x_{2}, x_{3}\right)$ of the velocity field:

$$
\left\{\begin{array}{l}
\dot{x}_{1}=x_{1}\left(\lambda+a x_{1}^{2}+b x_{2}^{2}+c x_{3}^{2}\right) \\
\dot{x}_{2}=x_{2}\left(\lambda+a x_{2}^{2}+b x_{3}^{2}+c x_{1}^{2}\right) \\
\dot{x}_{3}=x_{3}\left(\lambda+a x_{3}^{2}+b x_{1}^{2}+c x_{2}^{2}\right) .
\end{array}\right.
$$

By re-scaling $\left(x_{1}, x_{2}, x_{3}\right)$ and time we can fix

$$
\lambda=1 \text { and } a+b+c=-1 \text {. }
$$

Later on, Guckenheimer and Holmes [6] showed the existence of a structurally and asymptotically stable heteroclinic cycle in these equations provided that the coefficients $a, b$ and $c$ satisfy the conditions

$$
|a+b+c|=1 c<a<b<0 \text { and } 2 a>b+c .
$$

This heteroclinic cycle consists of six equilibrium points of saddle type, each lying on the coordinate axes connected by trajectories in the coordinates planes which are invariant by the flow corresponding to fixed point subspaces for the symmetry group $\Gamma=\mathbf{T} \oplus \mathbf{Z}_{2}$. Here $\mathbf{T}$ denotes the tetrahedral group and acts as a cyclic permutation. The group $\mathbf{Z}_{2}$ acts as a reflection. Roughly speaking, we have six equilibrium points each lying on the coordinate axes that are connected by trajectories in the invariant planes $x_{i}=0, i=1,2,3$ guaranteed by the Poincaré Bendixon Theorem (see Figure 1).

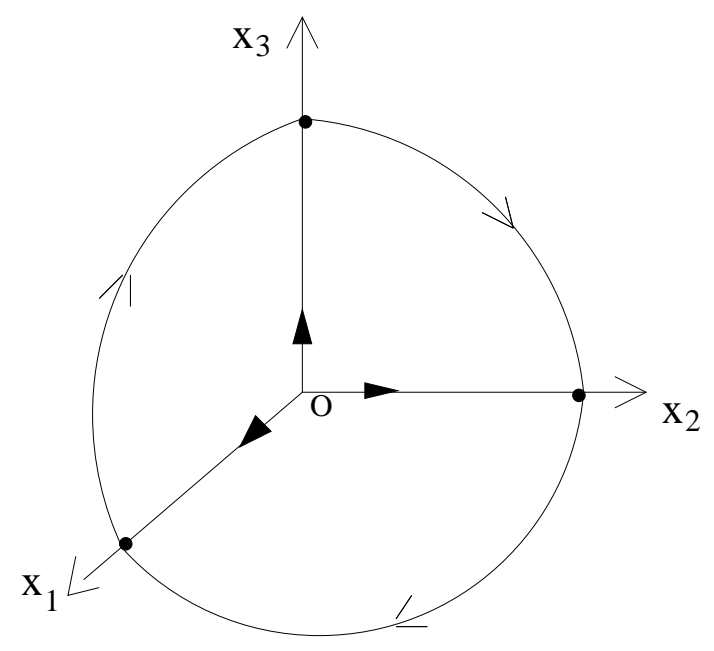

Figure 1. The heteroclinic cycle in $\mathbb{R}^{3}$ of Guckenheimer and Holmes.

Starting from system equations (1.2) we will focus our efforts on the study of chaotic dynamics in the singularly perturbed model (1.1). More specifically, we will study what is called a transverse perturbation of the heteroclinic cycle existing in system (1.2) and in our case, this perturbation will lead us to a singular system as $\varepsilon$ is a small parameter. The transverse perturbation consists of adding a fourth equation to the system (1.2) for a dynamic in a direction orthogonal to the original 
invariant coordinate planes. These kinds of perturbed systems have been studied by several authors within the context of symmetric dynamical systems. Their approach relies heavily on the equivariant bifurcation theory and symmetry group techniques (see Sandstede and Scheel [9], Melbourne and Krupa [7]).

In this paper we will consider a singular transverse perturbation and, for our purposes the symmetry considerations will play a minor role. At first the variable $u$ was meant to mimic the dynamics of a magnetic instability resulting from a convective state described by equations (1.2). The scale for the magnetic mode $u$ arises from the time scale of approach to the limit cycle of the fast dynamics in the velocity variables $x_{i}, i=1,2,3$. This model failed to be representative of the dynamo effect because of the symmetries of the equation in $u$. Since the magnetic field should respect the symmetry $u \mapsto-u$ only even powers of $u$ are allowed. This unfortunately does not exhibit rich dynamical behavior for this $u$ variable. Nevertheless, the work presented here is aimed to gain some insight into what kind of dynamics one can expect when breaking heteroclinic cycles in $\mathbb{R}^{3}$ that were forced by symmetry. The global behavior of the perturbed system (1.1) can be very complicated as we will show in the next section despite the apparently simple form of the equation for the $u$ variable. The terms in this equation have the following justification: $\mu<0$ since we require asymptotic stability, $p_{1}, p_{2}, p_{3}$ can be of any sign with $p_{3} \neq 0$ since the quantity $x_{1} x_{2} x_{3}$ plays a fundamental role as we will show later. The parameter $p_{1}$ is responsible for breaking the original heteroclinic connections. Finally, the equation for the variable $u$ respects some symmetry properties, notably $\left(x_{1}, x_{2}, x_{3}, u\right) \mapsto\left(-x_{1},-x_{2},-x_{3},-u\right)$ which simplifies the bifurcation analysis.

As it will become apparent from numerical simulations, chaotic orbits appear in the fourth dimensional system with a reminiscent behavior inherited from the three dimensional heteroclinic cycle of the system (1.2). In order to identify the origin of the chaos we observe that the motion has three components. The first one is the symmetry inherited from the slow equations. The second is the fact that the orbits have long residence times close to the heteroclinic cycles. Finally, an erratic dynamics is produced by the fast motion when the orbit goes from one slow manifold to the other. To determine the mechanism responsible for the dynamics we show that the equations have Hopf bifurcation points. Also, their bifurcation points lie on the slow manifolds which have a strongly attracting direction. Then these bifurcated orbits as the parameters change enter the attracting region of the heteroclinic cycles for the system. Due to this fact the orbits acquire a long time scale compared to the Hopf time. Since the attracting region overlaps the fold of the slow manifolds the orbits jump erratically from one manifold to the other. This is the reason for the appearance of chaotic orbits.

As a result a new $3 \mathrm{D}$ strange attractor forms in $\mathbb{R}^{4}$ whose structure resembles the structure of a $2 \mathrm{D}$ tent map. This invariant chaotic set is composed by a Cantorlike union of leaves and on each leaf the behavior is the same one as for the tent map. The mechanism for the creation of such an object is identified as the result of coupling two Hopf bifurcations in the slow manifolds with a fast jump process transverse to those manifolds. A more detailed analysis shows us that the creation of chaos comes from the situation where the slow manifolds loose normal hyperbolicity and present knees. The structure of these manifolds is inherited from the original heteroclinic connections. The drop points where the orbits jump from one slow manifold to another happen to be far enough from the Hopf equilibria. The 
geometric situation can be described as a 3 -dimensional figure $S$ whose geometry is the result of adding the new transverse direction to the original 1-dimensional heteroclinic connections.

The layout of the paper is as follows. In Section 2 we present the numerical simulations that induced us to search for chaos. Section 3 is devoted to a bifurcation analysis that allows us to derive a geometric model presented in Section 4 . In the singular limit case, $\varepsilon=0$, we can solve $u$ as a (bi-valuable) function of the slow variables $x_{1}, x_{2}, x_{3}$ and obtain a 3 -dimensional system of equations. It will be assumed that there exists a very strong contracting direction transverse to the slow manifolds that allows us to reduce the study of the dynamics to a two dimensional system of equations. The proof of the existence of chaos is tackled in Section 5 which is divided in two different parts. In a first sub-section we treat the singular limit case $(\varepsilon=0)$ for which we can exhibit a Poincaré map. This Poincaré map has a positive Lyapunov exponent. For $\varepsilon \neq 0$ it is unknown the explicit form of the Poincaré map. However we can prove that there exists at least an invariant curve where the dynamics are conjugated to those of a chaotic map. We will employ the $\mathcal{C}^{1}$-approximation theory developed by Mischenko et al. [8]. This is the content of the second sub-section closing the study of chaos and the paper.

\section{Numerical Simulations}

The system (1.1) has been numerically integrated using a Runge-Kutta scheme of order 7-8 with adjustable step. The typical phase portrait for an orbit is shown in Figure 2, where we have fixed $a=-0.2, b=-0.1, c=-0.7$ and $\varepsilon=0.01$. Without loss of generality these values will remain fixed for the remainder. Numerical simulations of such system present the following features: typical orbits approach the original heteroclinic connections but never asymptotically. After a number of revolutions in the neighborhood of the heteroclinic orbit, say in the first octant, they jump to another octant and repeat the process in a random fashion. At this stage, the chaotic behavior consists on the unpredictability of the time spent on each octant and the way these octants are visited by the orbit. These erratic excursions depend on the sign and amplitude of the term $p_{3} x_{1} x_{2} x_{3}$. A crucial consequence of this is the fast changes of sign in the $u$ variable as shown in figure 2 . Hence from figures 2 and 3 we can have a glimpse of the dynamics for the full system: the remains of the rupture of the original heteroclinic orbits which are clearly seen in figure 2, play the role of separatrices. The orbits spiral around a fixed point and are confined to a thin region close to the old heteroclinic orbits. While this process is occurring for the $x_{1}, x_{2}, x_{3}$ variables the variable $u$ suffers a dramatic oscillation in a much faster time scale. This will serve as the mold for the geometric model and the resulting attractor.

The numerical simulations presented in these two figures induce us to study the dynamics with the use of fast-slow techniques (see Section 4). Another observation is that as the parameters are varied, bifurcations take place so that there are slow manifolds (i.e. in the $x_{1}, x_{2}, x_{3}$ variables) that lose normal hyperbolicity. This must be reflected by the fact that eigenvalues associated to the behavior normal to these invariant planes cross zero. This bifurcation phenomenon will be detailed in Section 3.

To reinforce this evidence of chaotic behavior sensitive dependence on initial conditions has been observed in the computer; close initial conditions diverge into 


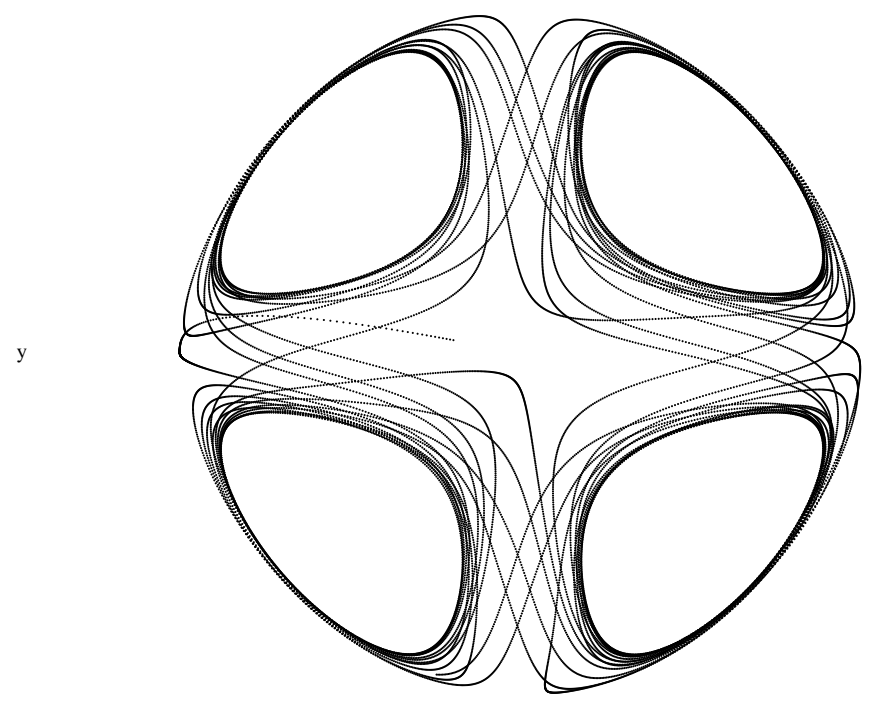

$\mathrm{x}$

Figure 2. Phase portrait in the $x_{1}, x_{2}$ space for $\mu=-0.05, p_{1}=$ $-0.07, p_{2}=0.5, p_{3}=0.8$ and $\varepsilon=0.01$.

completely different trajectories. Besides these erratic excursions, the jumps between the octants for the $x_{i}$ variables are accompanied by fast reversals of the sign of the variable $u$. The duration of the epochs will be shown to also be chaotic. 


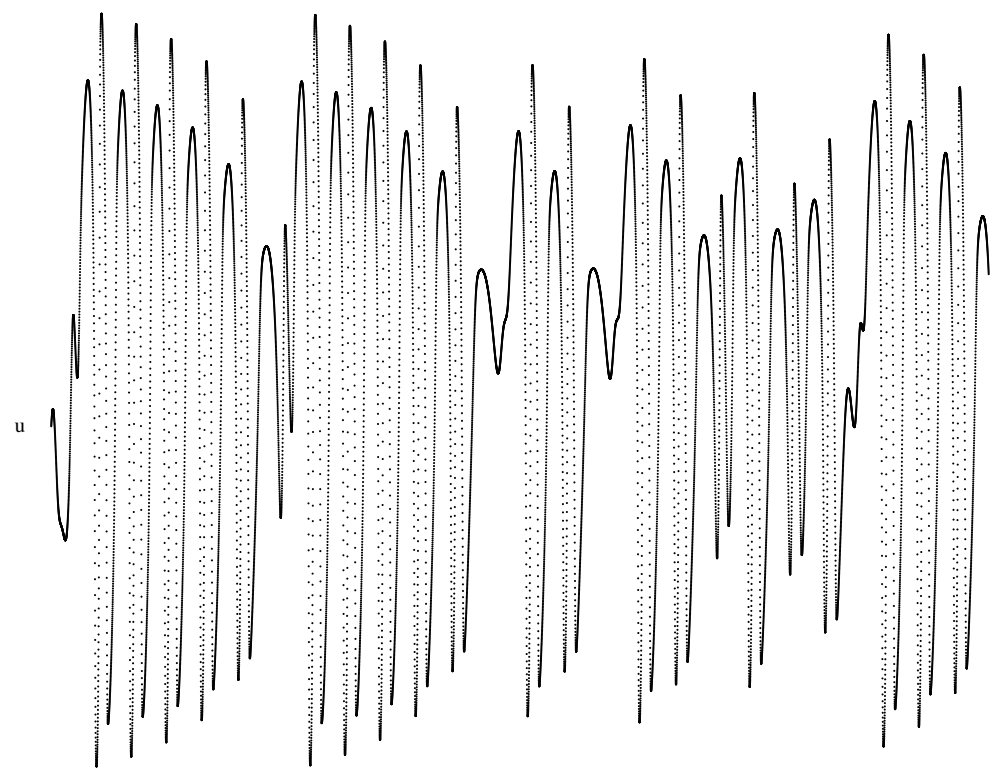

time

Figure 3. Chaotic behavior for the u variable.

Figure 4 shows a two dimensional projection of the Poincaré section $u=0$ for the system (1.1). This three dimensional object resembles a ribbon with a width that is folded at an angle and resembles the image of a tent. Most likely this strange attractor is composed of a union of infinitely many one-dimensional tents glued together in a transverse direction. Topologically this object could be the product 


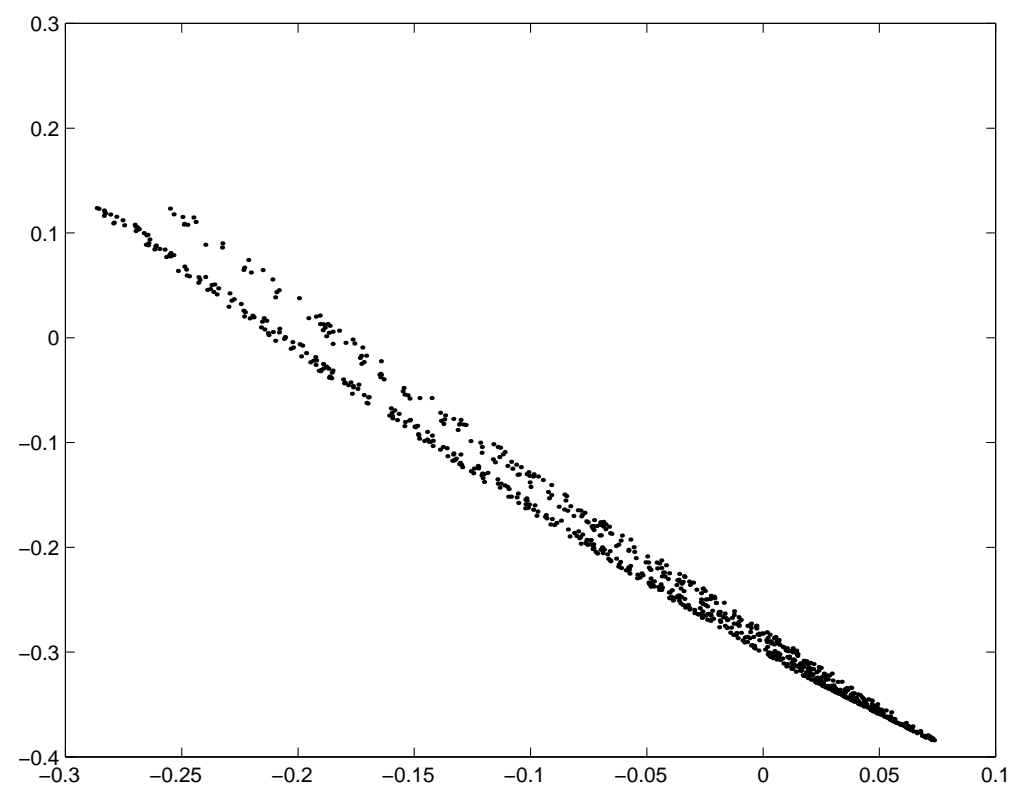

FiguRE 4. 2D projection of a 3D Poincaré section $u=0$ for the same parameter values as in Figure 2

of an interval together with a tent map. The dynamics along the tent maps are easily proved to be chaotic and the novelty of this new type of strange attractor comes from the fact that its width looks like an interval that could itself be a Cantor set.

Another numerical evidence for the presence of chaos is the existence of positive Lyapunov exponents that are consistent with the existence of a strange attractor. Starting from an infinitesimal four dimensional sphere of initial conditions this sphere deforms into a four dimensional ellipsoid due to the locally deforming nature of the flow. Then the $i_{t h}$ dimensional Lyapunov exponent is defined in terms of the length of the ellipsoidal principal axes $a_{i}(t)$. The effective computation of the Lyapunov exponents has been done for different values of the parameters $p_{i}$ starting from 10 different points chosen equally spaced on a circumference of radius 0.01 around the origin. We then computed the most positive exponent $L_{1}$ up to $\mathrm{n}=30000$ iterations:

$$
L_{1}=\max _{i} L_{i}=\lim _{t \rightarrow \infty} \log _{2} \frac{a_{i}(t)}{a_{i}(0)}
$$

The table below presents some parameter values where $L_{1}$ is representative. The fixed values are $\mu=-0.05$ and $\varepsilon=0.01$.

\section{Bifurcation anAlysis}

In this section we will prove the existence of the equilibrium points founded numerically in Section 2. More specifically the purpose is to understand the change in the orbit structure of the flow (1.1). Remember from Section 2 that there seemed to exist equilibrium points around which the orbits spirals. 


\begin{tabular}{|c|c|}
\hline Parameter values & Lyapunov Exponent \\
\hline \hline$p_{1}=0.53 p_{2}=0.1 p_{3}=-1.6$ & $L_{1}=0.017$ \\
\hline$p_{1}=0.23 p_{2}=-0.2 p_{3}=-1.6$ & $L_{1}=0.34$ \\
\hline$p_{1}=0.33 p_{2}=-0.1 p_{3}=-1.5$ & $L_{1}=0.72$ \\
\hline$p_{1}=0.73 p_{2}=0.3 p_{3}=-1.1$ & $L_{1}=0.69$ \\
\hline$p_{1}=1.03 p_{2}=0.6 p_{3}=-0.8$ & $L_{1}=4.45$ \\
\hline
\end{tabular}

TABLE 1. Lyapunov Exponents for system (1.1)

We will focus attention to a small neighborhood of those parameter values for which there is a non hyperbolic equilibrium point. As the parameters are varied, bifurcations take place in which the real part of the eigenvalues associated to the behavior normal to the invariant planes cross zero. Although the parameters $\mu$ and $\varepsilon$ can be fixed for once without lose of generality, the dependence on the parameters $p_{1}, p_{2}, p_{3}$ obliges us to use numerical methods together with analytical results.

Proposition 3.1. The non trivial equilibria for the system (1.1) are as follows:

(1) Type I: If $u=0$, then $E_{x^{ \pm}}=\left( \pm \sqrt{-\frac{1}{a}}, 0,0,0\right), E_{y^{ \pm}}=\left(0, \pm \sqrt{-\frac{1}{a}}, 0,0\right)$ $E_{z \pm}=\left(0,0, \pm \sqrt{-\frac{1}{a}}, 0\right)$ and $( \pm 1, \pm 1, \pm 1,0)$.

(2) Type II: If $u \neq 0$ and if $\exists x_{i}=0, i=1,2,3$ then $E_{X_{u}^{ \pm}}=\left( \pm \sqrt{-\frac{1}{a}}, 0,0\right.$, $\left.\pm \sqrt{-\mu+\frac{p_{2}}{a}}\right), E_{Y_{u}^{ \pm}}=\left(0, \pm \sqrt{-\frac{1}{a}}, 0, \pm \sqrt{-\mu+\frac{p_{2}}{a}}\right)$ $E_{Z_{u}^{ \pm}}=\left(0,0, \pm \sqrt{-\frac{1}{a}}, \pm \sqrt{-\mu+\frac{p_{2}}{a}}\right)$

(3) Type III: If $u \neq 0$ and $x_{1} x_{2} x_{3} \neq 0$ then $E_{U_{i}^{ \pm}}=\left(x^{*}, \pm x^{*}, \pm x^{*}, u^{*}\right), i=$ $1, \cdots, 4$.

For type III equilibria the signs can be chosen independently in each component. Remark also that due to the symmetries of the system

$$
\begin{aligned}
\left(x_{1}, x_{2}, x_{3}, u\right) & \mapsto\left(x_{2}, x_{3}, x_{1}, u\right) \\
\left(x_{1}, x_{2}, x_{3}, u\right) & \mapsto\left(-x_{1}, x_{2}, x_{3}, u\right) \\
\left(x_{1}, x_{2}, x_{3}, u\right) & \mapsto\left(-x_{1},-x_{2},-x_{3},-u\right),
\end{aligned}
$$

we can do all the bifurcation analysis in one octant. We will compute the eigenvalues only at the first octant $\left(x_{i}>0, i=1,2,3\right)$ since the others are easily obtained by cyclic permutation.

Proposition 3.2. (1) Type I equilibria are as follows:

(a) The equilibria $E_{x^{ \pm}}, E_{y^{ \pm}}, E_{z^{ \pm}}$are saddles except at the particular case $\mu=p_{2} / a$.

(b) The equilibria $( \pm 1, \pm 1, \pm 1,0)$ are sinks.

(2) Type II equilibria $E_{X_{u}^{ \pm}}, E_{Y_{u}^{ \pm}}, E_{Z_{u}^{ \pm}}$are saddles.

Proof. Statement 1 of proposition 3.2 comes from straightforward computations. At the equilibria type I the eigenvalues are:

$$
\lambda_{1}=-2 ; \quad \lambda_{2}=\left(1-\frac{b}{|a|}\right) ; \quad \lambda_{3}=\left(1-\frac{b}{|a|}\right) ; \quad \lambda_{4}=\frac{1}{\varepsilon}\left(\mu-\frac{p_{2}}{a}\right) .
$$


At the equilibria type II the eigenvalues are:

$$
\lambda_{1}=-2 ; \quad \lambda_{2,3}=-\frac{1}{2}\left(1-\frac{b}{|a|}\right)+\sqrt{\Delta} ; \quad \lambda_{4}=\frac{4}{\varepsilon}\left(\mu-\frac{p_{2}}{a}\right)
$$

where $\Delta=\left(1+\frac{b}{a}\right)^{2}+4\left(p_{1}^{2} x_{1}^{2} u^{2}+2\left(1-\frac{b}{a}\right)\right)$ is always positive. Hence for any value of the parameters $\mu, \varepsilon, p_{1}$ and $p_{2}$ (recall that $a, b, c$ satisfy conditions (1.3)) all these eigenvalues are real.

This result was to be expected from the numerical simulations done in Section 2 that clearly show a dramatic change of dynamics close to equilibria type III located in the axis.

Proposition 3.3. A Hopf bifurcation occurs at type III equilibria.

This last proposition deserve special attention since this Hopf bifurcation is a key point in the understanding of the construction of the geometric model in Section 4. The equilibrium point $E_{U_{i}^{ \pm}}$satisfy the equation :

$$
\left(x_{1}^{2}-x_{2}^{2}\right)+a\left(x_{1}^{4}-x_{2}^{4}\right)+b x_{2}^{2}\left(x_{1}^{2}-x_{3}^{2}\right)+c x_{1}^{2}\left(x_{3}^{2}-x_{2}^{2}\right)=0
$$

whose solutions are $x_{1}= \pm x_{2}= \pm x_{3}=x^{*}$. Replacing terms, system (1.1) reads:

$$
\left\{\begin{array}{c}
1-x^{*^{2}}+p_{1} x^{*} u=0 \\
u\left(\mu-u^{2}\right)+3 p_{2} x^{*^{2}} u+p_{3} x^{*^{3}}=0 .
\end{array}\right.
$$

The eigenvalues at the equilibria type III can only be computed numerically. Of course the eigenvalues of the linearization depend also on $\varepsilon$. The analytical expression of the polynomial function the eigenvalues satisfy is of no use because of its complexity. Instead we will fix $\varepsilon=0.01$ (as well as $a, b$ and $c$ ) and compute numerically these eigenvalues varying $p_{1}, p_{2}, p_{3}$. More concretely, we first solve from $1-x^{*^{2}}+p_{1} x^{*} u=0$ obtaining $x^{*}=f(u)$ and then we replace its values into the second equation. In this way we get an equation of the form $F(u)=0$ that we solve numerically. Once these numerical solutions $u=u^{*}$ have been found we have all the numerical data to compute the eigenvalues numerically as well.

In table 3.2 we present some values proving the occurrence of Hopf bifurcations along the axis $x_{1}= \pm x_{2}= \pm x_{3}=x^{*}$.

A proof that the Hopf bifurcation takes place on the slow manifold is as follows: for one of the slow variables $x_{i}, i=1,2,3$, say $x_{1}$ large, take $u=\beta x_{1}$ for the approximated root. This will lie in the slow manifold $|u| \geq\left(\frac{u+3 x_{1}^{2}}{3}\right)^{1 / 2}$ provided $\beta>1$. To the leading order the equation for $\beta$ is

$$
-\beta^{3} x_{1}^{3}+3 p_{2} \beta^{2} x_{1}^{3}=p_{3} x_{1}^{3},
$$

that is $\beta^{3}-3 p_{2} \beta^{2}=p_{3}$ since $x_{1} \neq 0$. Hence for $p_{3}$ large we will have $\beta>1$. The equation for $x_{1}$ now reads $1-x_{1}^{2}+\beta p_{1} x_{1}^{2}=0$ that is

$$
x_{1}^{2}\left(1-\beta p_{1}\right)=1 \text {. }
$$

Thus if $\beta p_{1} \simeq 1$ we have that $x_{1}$ is large and the Hopf point lies in the slow manifold.

It is important to remark that the dynamics in the variable $u$ are always bounded. In fact, let us denote

$$
\Pi\left(x_{1}, x_{2}, x_{3}, u\right)=u\left(\mu-u^{2}+p_{2}\left(x_{1}^{2}+x_{2}^{2}+x_{3}^{2}\right)\right)+p_{3} x_{1} x_{2} x_{3} .
$$


Observe that when $x_{1} x_{2} x_{3}=0$, the roots $\tilde{r}_{1,2}= \pm \sqrt{\mu+p_{2}\left(x_{1}^{2}+x_{2}^{2}+x_{3}^{2}\right)}$ and $\tilde{r}_{3}=0$, are such that $D_{u} \Pi\left(u, x_{1}, x_{2}, x_{3}\right)=\mu+p_{2}\left(x_{1}^{2}+x_{2}^{2}+x_{3}^{2}\right)>0$ at $\tilde{r}_{3}$ while at $\tilde{r}_{1}$ and $\tilde{r}_{2} D_{u} \Pi\left(u, x_{1}, x_{2}, x_{3}\right)=-2\left(\mu+p_{2}\left(x_{1}^{2}+x_{2}^{2}+x_{3}^{2}\right)\right)<0$. By structural stability of these hyperbolic points, that is, as long as the roots do not collapse, and except at the particular point where the hyper plane $x_{1} x_{2} x_{3}=0$ is tangent to $D_{u} \Pi\left(x_{1}, x_{2}, x_{3}, u\right)=0$, we can conclude that for $x_{1} x_{2} x_{3} \neq 0$ the variable $u$ also remains bounded and two stable roots $r_{1}, r_{2}$ persist.

We end this section with two important propositions.

Proposition 3.4. [6] Consider the system equation (1.2) under conditions (1.3). Then the closed spherical shell $\mathcal{D}$ with boundaries at radii $\sqrt{3}$ and $\sqrt{-\frac{1}{a}}$ is forward invariant under the flow and attracts all non-zero trajectories. All trajectories off the invariant lines $x_{1}= \pm x_{2}= \pm x_{3}$ approach the surface $x_{1} x_{2} x_{3}=0$.

The following proposition shows that if an orbit starts in $\left|x_{1} x_{2} x_{3}\right|<\delta$ that is sufficiently close to the old surface $x_{1} x_{2} x_{3}=0$ it will be trapped in a thin shell $0 \leq K \leq\left(x_{1}^{2}+x_{2}^{2}+x_{3}^{2}\right) \leq R$ and remain there. This is in perfect agreement with the numerical simulations in Section 2.

Proposition 3.5. [1] There exists $0<K(\delta)<R(\delta)$ such that for any initial data satisfying $\left|x_{1} x_{2} x_{3}\right|<\delta$ the region $0<K \leq\left(x_{1}^{2}+x_{2}^{2}+x_{3}^{2}\right) \leq R$ is invariant and attracting. Moreover, as $\delta \longrightarrow 0$,

$$
\begin{gathered}
K(\delta) \longrightarrow-\frac{2}{b+c} \\
R(\delta) \longrightarrow-\frac{1}{a} .
\end{gathered}
$$

\section{The Geometric MOdeL}

The numerical simulations presented in Section 2 can now be interpreted as follows: the trajectories spiral away from the Hopf points (equilibria type III) determined in Section 3 and are attracted into the thin shell of proposition [3.4). This region overlaps the kink region of the slow manifold. Thus the solution undergoes a fast jump process. This scenario is repeated infinitely as time goes by. It is to be noted that the jump does not depend on the existence of a heteroclinic connection. It rather depends on the large amplitude of the limit cycle surrounding the Hopf equilibrium. This limit cycle arising as a continuation of the bifurcated one overlaps the kink of the slow manifold. Because of this, we can formulate a simple model in terms of two Hopf bifurcations coupled with a fast transition. We would like to emphasize the fact that the geometric model obtained in this section can be understood in our approach only by considering that due to the strongly attractive dynamic in one direction, the phenomenon is essentially three dimensional. This reduction is justified by the fact that $\lambda_{1}, \lambda_{2}<0$ and, of course, is just an approximated model. With these considerations in mind we can now describe some of the features observed in the numerical simulations.

The time scale for $u$ arises from the time scale of approach to the limit cycle. During that period the variable $u$ moves in a fast manifold and oscillates i.e. changes sign very rapidly. After that, the orbit passes close to an equilibrium point of type 


\begin{tabular}{|c|c|c|c|c|c|c|c|}
\hline $\mathrm{p}_{1}$ & $\mathrm{p}_{2}$ & $\mathrm{p}_{3}$ & $\mathrm{x}^{*}$ & $\mathrm{u}^{*}$ & $\lambda_{1}$ & $\lambda_{2}$ & $\lambda_{3}, \lambda_{4}$ \\
\hline \hline-0.7 & 0.01 & -0.07 & 1.9305602 & 0.4819866 & -70.095331 & -1.9305602 & $-0.2385182 \pm 1.4536030 \mathrm{i}$ \\
\hline-0.7 & 0.02 & -0.07 & 1.1975489 & 0.5178712 & -77.653007 & -1.9308681 & $-0.2945972 \pm 1.4903846 \mathrm{i}$ \\
\hline-0.7 & 0.01 & -0.05 & 1.1571989 & 0.4186331 & -53.976178 & -1.9217082 & $-0.1425747 \pm 1.3916433 \mathrm{i}$ \\
\hline-0.7 & 0.01 & -0.01 & 1.0761981 & 0.2600015 & -15.059158 & -1.8546287 & $0.1468763 \pm 1.2036391 \mathrm{i}$ \\
\hline-0.7 & 0.01 & -0.02 & 1.1047107 & 0.2849946 & -26.035848 & -1.8899715 & $0.0473829 \pm 1.2682620 \mathrm{i}$ \\
\hline-0.5 & 0.01 & -0.07 & 1.1194786 & 0.4524088 & -62.921553 & -1.9501396 & $-0.005169 \pm 1.3023957 \mathrm{i}$ \\
\hline-0.3 & 0.01 & -0.07 & 1.0661523 & 0.4273330 & -56.540715 & -1.9699277 & $0.1813103 \pm 1.1812732 \mathrm{i}$ \\
\hline-0.05 & 0.01 & -0.07 & 1.0100691 & 0.4007570 & -50.146429 & -1.9949555 & $0.3676166 \pm 1.0602641 \mathrm{i}$ \\
\hline
\end{tabular}

TABLE 2. Numerical values of some eigenvalues showing the existence of a Hopf bifurcation at equilibrium points of type III.

II and joins a slow manifold where $u$ slowly increases (decreases) until a critical value that corresponds to a point in the upper (lower) branch, depending on the value and sign of the quantity $x_{1} x_{2} x_{3}$.

By replacing the variable $u$ by either one of the stable roots $r_{1,3}$ of the polynomial $\Pi\left(x_{1}, x_{2}, x_{3}, u\right)$ we obtain for $\varepsilon=0$ a singular system having solutions which exhibit jumps. Let $u_{1}=\varphi_{1}\left(x_{1}, x_{2}, x_{3}\right)$ and $u_{2}=\varphi_{2}\left(x_{1}, x_{2}, x_{3}\right)$ denote the slow manifolds. Substituting the expression for $u$ into the system (1.1) we obtain:

$$
\left\{\begin{array}{l}
\dot{x}_{1}=x_{1}\left(1+a x_{1}^{2}+b x_{2}^{2}+c x_{3}^{2}\right)+p_{1} x_{2} x_{3} \varphi_{i}\left(x_{1}, x_{2}, x_{3}\right) \\
\dot{x}_{2}=x_{2}\left(1+a x_{2}^{2}+b x_{3}^{2}+c x_{1}^{2}\right)+p_{1} x_{1} x_{3} \varphi_{i}\left(x_{1}, x_{2}, x_{3}\right) \quad i=1,2 . \\
\dot{x}_{3}=x_{3}\left(1+a x_{3}^{2}+b x_{1}^{2}+c x_{2}^{2}\right)+p_{1} x_{1} x_{2} \varphi_{i}\left(x_{1}, x_{2}, x_{3}\right)
\end{array} \quad i=\right.
$$

By virtue of the center manifold theorem for a Hopf bifurcation, we can write the system above into the following normal form:

$$
\left(\begin{array}{c}
\dot{x} \\
\dot{y} \\
\dot{z}
\end{array}\right)=\left(\begin{array}{ccc}
\alpha(u) & -\beta(u) & 0 \\
\beta(u) & \alpha(u) & 0 \\
0 & 0 & \varrho(u)
\end{array}\right)\left(\begin{array}{l}
x \\
y \\
z
\end{array}\right)+\left(\begin{array}{c}
P\left(x, y, z ; p_{1}\right) \\
Q\left(x, y, z ; p_{1}\right) \\
R\left(x, y, z ; p_{1}\right)
\end{array}\right),
$$

where $P, Q, R$ are nonlinear terms which vanish at the equilibria. The parameter $p_{1}$ controls the local bifurcation assumed to occur at $\varrho\left(u_{c}\right)=0$ and be super-critical; for $u \neq u_{c}$, we have $\varrho(u)$ negative and $\alpha(u)$ positive with $\alpha(u)>|\varrho(u)|$ with the 
jump conditions imposed on the surfaces of double roots. The perturbation theory for singularly perturbed guarantees the persistence of singular orbits when $\varepsilon \neq 0$. However it is difficult to obtain explicit information from the Poincare map due to the 3 -dimensional nature of the system. In our case since $\varrho\left(u_{1,2}\right)$ are negative we have a strong contracting direction and we can use the center manifold reduction for both systems and reduce the study of a two dimensional system which takes the form:

$$
\left(\begin{array}{c}
\tilde{x} \\
\tilde{y}
\end{array}\right)=\left(\begin{array}{cc}
\alpha(u) & -\beta(u) \\
\beta(u) & \alpha(u)
\end{array}\right)\left(\begin{array}{c}
\tilde{x} \\
\tilde{y}
\end{array}\right)+\left(\begin{array}{c}
\tilde{P} \\
\tilde{Q}
\end{array}\right),
$$

together with the matching conditions on the center manifold $\tilde{z}=\Xi(\tilde{x}, \tilde{y})$ along curves where $\varphi_{1}(\tilde{x}, \tilde{y})=\varphi_{2}(\tilde{x}, \tilde{y})$. An appropriate set of parameter values exists such that this center manifold intersects with the drop point. In what follows we denote $X=(\tilde{x}, \tilde{y})$. The model system takes the form:

$$
\left\{\begin{array}{c}
\dot{X}=F(X, u) \\
\varepsilon \dot{u}=\left(u-r_{1}(X)\right)\left(u-r_{2}(X)\right)\left(u-r_{3}(X)\right) .
\end{array}\right.
$$

The singular system is thus

$$
\dot{X}=F\left(X, \varphi_{1,2}(X)\right)
$$

with the continuity condition along the curves of double roots $\mathrm{r}_{1,2}(X)=r_{3}(X)$.

It follows from section 3 that on the slow manifolds $r_{1,2}$, the flow undergoes a Hopf bifurcation at the points $u=u_{1}$ and $u=u_{2}$. Moreover, there is an attracting center manifold at both points where the bifurcation takes place. Initial conditions starting near the $r_{1}$-manifold are attracted to the shell. The center manifold where the solution lies intersects with the drop point on the $r_{1}$-manifold. At this point the solution jumps to the $r_{2}$-branch and this process is repeated infinitely many times. As the parameter regime is changed the only amplitude effect is the possibility of jumping between different octants in the $r_{1}$ or $r_{2}$-manifold.

There is another situation when the parameters are such that the attracting shell $\mathcal{D}$ never intersects with the drop point of the $r_{1,2}$-manifold. In such a case the motion is constrained either to $r_{1}$ or to $r_{2}$. The Hopf bifurcated orbit can be continued to finite amplitude and all solutions tend asymptotically to a periodic orbit. This case has been studied as a perturbation of the heteroclinic cycle for the simple case of a quadratic equation for the variable $u$, where there is only one stable root (see [3]). We also remark that systems presenting a switch have been studied numerically by Deng [4] in the singular limit case.

\section{The Poincaré MAPS}

In this section we show that the Poincaré map associated to the geometric model derived is indeed a map possessing chaotic orbits. As shown in he previous section we have a normal form for a coupled Hopf bifurcation that mimics the behavior observed for our four dimensional original model. We also showed in the bifurcation analysis section that there is always a strong contraction in one of the directions. What we do next is to write down the explicit form of the Poincaré map by following the geometric model and prove that it has a positive Lyapunov exponent. 
5.1. The Poincaré map for $\varepsilon=0$. The Poincaré map we construct is the composition of two different maps $\varphi^{i}$ and $\psi^{i}$. The auxiliary maps $\varphi^{i}$ are defined by the flow connecting two slow manifolds. This map, for $\varepsilon=0$, is just a projection. We could take a more general connecting diffeomorphism but it would not introduce any qualitative changes in the dynamics we are interested. The second maps $\psi^{i}$ are defined by the flow near the equilibria and their expression is valid only away from the break section where the jumps occur. The geometric construction presented in this subsection follows the geometric model presented in Section 4. By an abuse of notation we will denote $X=(\tilde{x}, \tilde{y})=(x, y)$.

We place two planar surfaces $\Sigma_{i}, i=1,2$; each one in a neighborhood of an equilibrium point of type III and transverse to the corresponding unstable manifold:

$$
\begin{gathered}
\Sigma_{1}=\left\{(x, y, z): 0<y, z=z_{1}\right\} \\
\Sigma_{2}=\left\{(x, y, z): 0<y, z=z_{2}\right\} .
\end{gathered}
$$

Next we define the secants

$$
\begin{gathered}
S_{1}=\left\{(x, y, z) \in \Sigma_{1}, x=0\right\} \\
S_{2}=\left\{(x, y, z) \in \Sigma_{2}, x=A=\text { Const } .\right\} .
\end{gathered}
$$

We shall construct the one dimensional Poincaré map $\Pi_{0}: S_{1} \rightarrow S_{1}$ as the composition of the maps $\Psi_{0}^{1,2}: S_{1,2} \rightarrow S_{1,2}$ and $\Phi_{0}^{1}: S_{1} \rightarrow \Sigma_{2}, \Phi_{0}^{2}: S_{2} \rightarrow \Sigma_{1}$, that is $\Pi_{0}=\Psi_{0}^{1} \circ \Phi_{0}^{2} \circ \Psi_{0}^{2} \circ \Phi_{0}^{1}$ where

$$
\Psi_{0}^{i}(x, y)=\left(\begin{array}{l}
x e^{\mu_{i} t} \cos \omega_{i} t-y e^{\mu_{i} t} \sin \omega_{i} t \\
x e^{\mu_{i} t} \sin \omega_{i} t+y e^{\mu_{i} t} \cos \omega_{i} t
\end{array}\right) i=1,2,
$$

$\Phi_{0}^{1}(x, y)=(x, y+c)$ and $\Phi_{0}^{2}(x, y)=(x, y)$. Here $\omega_{i}$ denotes the frequency of the oscillation given by the imaginary part of the eigenvalues which depend continuously on $u$. Remark that we have taken for the maps $\Phi_{0}^{i}$ simple projections where $c$ denotes the offset of the equilibria.

We take as an initial point

$$
\left(\begin{array}{l}
x_{0} \\
y_{0} \\
z_{0}
\end{array}\right)=X_{u}^{1}=\left(\begin{array}{c}
0 \\
y \\
z_{1}
\end{array}\right) .
$$

Next point is $X_{l}^{1}, X_{u}^{1} \stackrel{\Phi_{0}^{1}}{\longrightarrow} X_{l}^{1} \in \Sigma_{2}$

$$
\begin{gathered}
\left(\begin{array}{l}
x_{1} \\
y_{1} \\
z_{1}
\end{array}\right)=\left(\begin{array}{c}
0 \\
y_{1}+c \\
z_{2}
\end{array}\right) \\
X_{l}^{1} \in \Sigma_{2} \stackrel{\Psi_{0}^{2}}{\longrightarrow} X_{l}^{2} \in S_{2} \\
\left(\begin{array}{c}
x_{2} \\
y_{2} \\
z_{2}
\end{array}\right)=\left(\begin{array}{c}
-(y+c) e^{\mu_{2} t^{(2)} \sin \omega_{2} t^{(2)}} \\
(y+c) e^{\mu_{2} t^{(2)} \cos \omega_{2} t^{(2)}} \\
z_{2}
\end{array}\right) \\
X_{l}^{2} \in S_{2} \stackrel{\Phi_{0}^{2}}{\longrightarrow} X_{u}^{2} \in \Sigma_{1}
\end{gathered}
$$




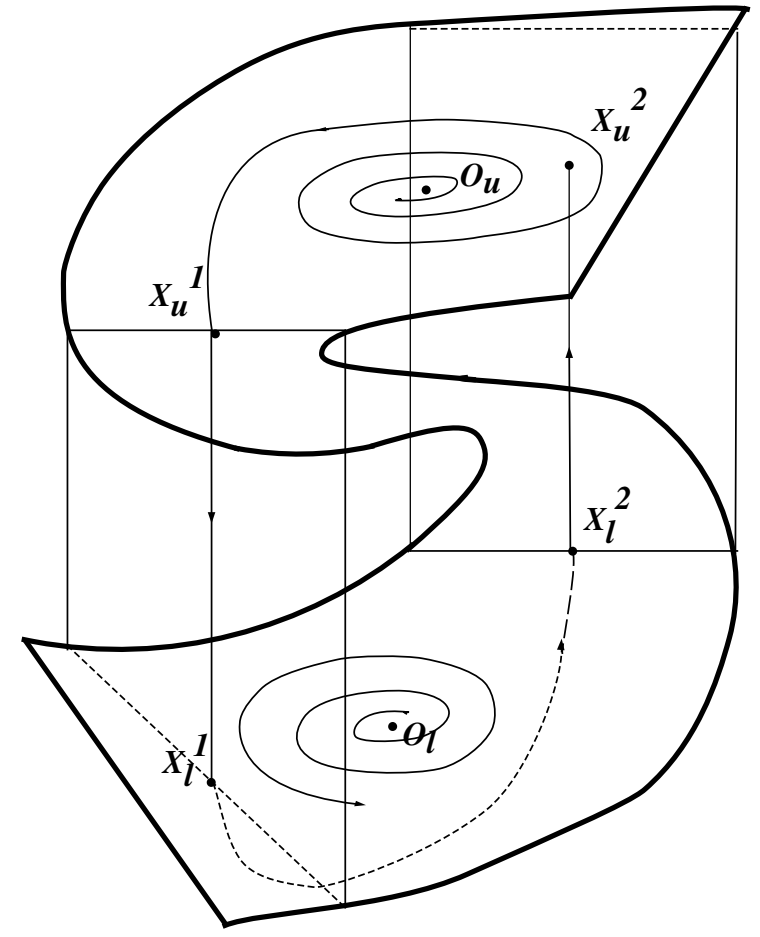

Figure 5. The geometric construction for the Poincaré map.

$$
\left(\begin{array}{l}
x_{3} \\
y_{3} \\
z_{3}
\end{array}\right)=\left(\begin{array}{c}
-(y+c) e^{\mu_{2} t^{(2)} \sin \omega_{2} t^{(2)}} \\
(y+c) e^{\mu_{2} t^{(2)} \cos \omega_{2} t^{(2)}} \\
z_{1}
\end{array}\right)
$$

and finally $X_{u}^{2} \in \Sigma_{1} \stackrel{\Psi_{0}^{1}}{\longrightarrow} \bar{X}_{u}^{1} \in S_{1}$

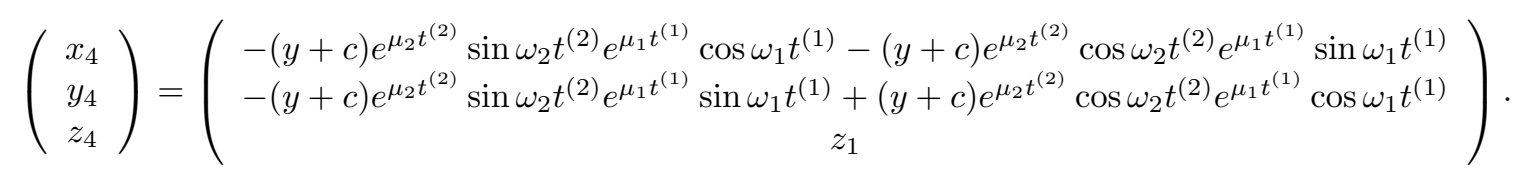

We have obtained the equations:

$$
\left\{\begin{array}{c}
0=-(y+c) e^{\mu_{2} t^{(2)}} \sin \omega_{2} t^{(2)} e^{\mu_{1} t^{(1)}} \cos \omega_{1} t^{(1)}-(y+c) e^{\mu_{2} t^{(2)}} \cos \omega_{2} t^{(2)} e^{\mu_{1} t^{(1)}} \sin \omega_{1} t^{(1)} \\
F_{0}(y)=-(y+c) e^{\mu_{2} t^{(2)}} \sin \omega_{2} t^{(2)} e^{\mu_{1} t^{(1)}} \sin \omega_{1} t^{(1)}+(y+c) e^{\mu_{2} t^{(2)}} \cos \omega_{2} t^{(2)} e^{\mu_{1} t^{(1)}} \cos \omega_{1} t^{(1)} .
\end{array}\right.
$$

We have denoted $t^{(2)}$ i the time given by the jump condition $A=(y+c) e^{\mu_{2} t^{(2)}} \sin \omega_{2} t^{(2)}$ and $t^{(1)}$ the time the orbit spends in the surface $\Sigma_{1}$. Solving the equations we find that $F_{0}(y)=(y+c) e^{\mu_{2} t^{(2)}+\mu_{1} t^{(1)}} \cos \left(\omega_{1} t^{(1)}+\omega_{2} t^{(2)}\right)$ together with the relationship between the times flight: $\sin \left(\omega_{2} t^{(2)}+\omega_{1} t^{(1)}\right)=0$. 
The Poincaré map is hence given by

$$
\left\{\begin{array}{c}
F_{0}(y)=(y+c) e^{\Omega t^{(2)}} \\
A=(y+c) e^{\mu_{2} t^{(2)}} \sin \omega_{2} t^{(2)}
\end{array}\right.
$$

where $\Omega=\frac{1}{\omega_{1}}\left(\omega_{1} \mu_{2}-\omega_{2} \mu_{1}\right)$.

Proposition 5.1. There exists a positive Lebesgue measure set $\mathcal{P}$ of parameter values such that for every $\Omega>0 \in \mathcal{P}$ and almost all $y \in I$;

$$
\lim _{n \rightarrow \infty} \sup \frac{1}{n} \log \left|\left(F_{0}^{n}\right)^{\prime}(y)\right|>0 \text {. }
$$

Proof. For the n-th iterated map $F_{0}^{n}$ we will denote $t^{(2 n)}$ the time the orbits spirals in the upper manifold $\Sigma_{2}$ before it reaches the drop line $S_{2}$. Thanks to the Implicit Function Theorem from the stopping conditions $A=(y+c) e^{\mu_{(2)} t^{(2 n)}} \sin \left(\omega_{2} t^{(2 n)}\right)$ we can write $t^{(2 i)}=g\left(A, F_{0}^{i}(y)\right)$ for all $1 \leq i \leq n$. For simplicity we will denote $t^{(2 k)}=g \circ F_{0}^{k}(\hat{y})$ and $(y+c)=\hat{y}$. We have

$$
F_{0}^{n}(\hat{y})=\hat{y} e^{\Omega \sum_{k=0}^{n-1} g \circ F_{0}^{k}(\hat{y})}
$$

together with the condition $\sum_{i=1}^{n} \omega_{1} t^{(i)}+\omega_{2} t^{(2 i)}=0$. Compute that

$$
\left(F_{0}^{n}\right)^{\prime}(\hat{y})=\left[1+\Omega(\hat{y}) \sum_{k=0}^{n-1} g^{\prime}\left(F_{0}^{k}(\hat{y})\right) \prod_{j=0}^{k-1} F_{0}^{\prime}\left(F_{0}^{j}(\hat{y})\right)\right] e^{\Omega \sum_{j=0}^{k-1} g \circ F_{0}^{j}(\hat{y})}
$$

We will assume the following hypothesis.

Hypothesis 1: There exists $\tau=\min _{i}\left\{t^{(2 i)}, t^{(i)}\right\}<\infty$. Note that this condition is automatically fulfilled from the moment we assume that there is at least the orbit jumping between the two slow manifolds.

Hypothesis 2: For each $1 \leq k \leq n$ there exists $M_{k}<-\infty$ such that

$$
0>\mu_{2}+\omega_{2} \cot \left(\omega_{2} g\left(F_{0}^{k}(\hat{y})\right)>M_{k} .\right.
$$

Let us denote $B=\left\{\hat{y} \in I=[0,1], \exists k_{0}, 0<k_{0}<\infty ; g^{\prime}\left(F_{0}^{k_{0}}\right)<0\right\}$.

We will assume that $m(B)=0$. This is equivalent to state that the set $B$ is a small set and for almost all $\hat{y} \in I \backslash B$ we have that $g^{\prime}\left(F_{0}^{k}(\hat{y})\right) \geq 0$ for all $k$. Physically this means that the further away from the unstable focus the return point hits the slow manifolds, the longer the time it takes to reach the drop sections. Assuming hypothesis 2 we have

$$
g^{\prime}\left(F_{0}^{k}(\hat{y})\right)=\frac{-1}{F_{0}^{k}(\hat{y})\left[\mu_{2}+\omega_{2} \cot \left(\omega_{2} g\left(F_{0}^{k}(\hat{y})\right)\right]\right.}>0
$$

Observe that

$$
F_{0}^{\prime}\left(F_{0}^{j}(\hat{y})\right)=\left[1+F_{0}^{j}(\hat{y}) \Omega g^{\prime}\left(F_{0}^{j}(\hat{y})\right] e^{\Omega g \circ F_{0}^{j}(\hat{y})}\right.
$$

which is strictly positive for $\hat{y} \in I \backslash B$. Now,

$$
\log \left|\left(F_{0}^{n}\right)^{\prime}(\hat{y})\right|=\Omega\left|\sum_{k=0}^{n-1} g \circ F_{0}^{k}(\hat{y})\right|+\log \left|1+\Omega \hat{y} \sum_{k=0}^{n-1} g^{\prime}\left(F_{0}^{k}(\hat{y})\right) \prod_{j=0}^{k-1} F_{0}^{\prime}\left(F_{0}^{j}(\hat{y})\right)\right|
$$


Thanks to hypothesis 1 we have $\Omega \sum_{k=0}^{n-1} g \circ F_{0}^{k}(\hat{y})>\Omega(n-1) \tau$. The second term can be estimated as follows:

From $(5.8)$ we have $F_{0}^{\prime}\left(F_{0}^{j}(\hat{y})\right)>\left(1-\Omega\left(M_{j}\right)^{-1}\right) e^{\Omega j \tau}$ hence replacing terms we get

$$
\begin{aligned}
\left.\sum_{k=0}^{n-1} g^{\prime}\left(F_{0}^{k}(\hat{y})\right) \Pi_{j=0}^{k-1} F_{0}^{\prime}\left(F_{0}^{j}(\hat{y})\right)\right] & =\sum_{k=0}^{n-1}\left(-M_{k}\right)^{-1} e^{-\Omega \tau} \prod_{j=0}^{k-1}\left(1+\Omega\left(-M_{j}\right)^{-1}\right) e^{\Omega j \tau} \\
& \geq \sum_{k=0}^{n-1}\left(-M_{k}\right)^{-1} e^{(k-2) \Omega \tau} \prod_{j=0}^{k-1}\left(1+\Omega\left(-M_{j}\right)^{-1}\right. \\
& \geq 0,
\end{aligned}
$$

which is sufficient to guaranty that

$$
\lim _{n \rightarrow \infty} \sup \left[\frac { 1 } { n } \left(\Omega(n-1) \tau+\log \left(1+\sum_{k=0}^{n-1}\left(-M_{k}\right)^{-1} e^{(k-2) \Omega \tau} \prod_{j=0}^{k-1}\left(1+\Omega\left(-M_{j}\right)^{-1}\right)\right]\right.\right.
$$

is for $\Omega>0$ a positive and finite number; the Lyapunov exponent.

5.2. Existence of an invariant curve for $\varepsilon \neq 0$. So far we have demonstrated that the dynamics of the system 1.1 can be reduced to the those of a map that possesses a positive Lyapunov exponent hence chaotic orbits when $\varepsilon=0$. Starting from the Poincaré map derived in the previous section, we can obtain the corresponding Poincaré map for $\varepsilon \neq 0$. The basic tool for this study is the $\mathbf{C}^{1}$-approximation theory developed by Mischenko et al. [8] concerning the Poincaré maps for singularly perturbed systems.

It is shown in [8] that one can choose a plane in the space $(X, u)$ which is transverse to the flow with coordinate $s$ along the stable manifold, say $u=\varphi_{1}(X)$ and coordinate $t$ along the transverse manifold. We have the following theorem:

Theorem 5.2 ([8]). The Poincaré map is given by

$$
\mathfrak{S}(s, t)=\left\{\begin{array}{l}
s \rightarrow F(s, t)=F_{0}(s)+\varepsilon^{2 / 3} \tilde{F}_{\varepsilon}(s, t) \\
t \rightarrow e^{-1 / \varepsilon} G(s, t)
\end{array}\right.
$$

The maps $F_{\varepsilon}, G_{\varepsilon}$ are continuously differentiable with respect $s, t$ and their derivatives are uniformly bounded with respect to $\varepsilon$ in the $\mathcal{C}^{1}$-metric.

The first coordinate is the map for the singular system perturbed by the map of the jump points which is of order $\mathcal{O}\left(\varepsilon^{2 / 3}\right)$ due to the scaling of the time in this region. The exponential contraction in the second coordinate is due to the exponential attractive character of the manifolds $u=\varphi_{1,2}(X)$. Notice that the estimates of Theorem (5.2) are uniform at the turning point since the equation for the integral manifold at the drop points is a Ricatti equation (see [8]). This equation is linear rigid as an Airy equation and introduces the factor $\varepsilon^{2 / 3}$ scaling at the drop fold points. The uniformity of the estimates is the content of Theorem (4.2) in [8].

We believe that this map possesses chaotic orbits but were unable to prove it rigorously. Instead we will show the persistence of an invariant curve which is sufficiently smooth and can be expressed in the form $t=\gamma(s)$. This curve lives in 
the three dimensional object presented in Figure 2 and is part of the skeleton of a much more complicated strange attractor.

Consider the rectangle $\mathbf{R}=\mathcal{I}_{s} \times \mathcal{I}_{t}$. For the following we re-parametrize $\mathcal{I}_{s}=$ $[0,1]$ and $\mathcal{I}_{t}=\left[-t_{0}, t_{0}\right]$. Let us denote the disk $\gamma_{0}=t_{0}^{*} \times \mathcal{I}_{s}, t_{0}^{*} \in \mathcal{I}_{t}$. Clearly this straight line is mapped inside the rectangle $\mathbf{R}$ since $\mathfrak{S}$ strongly contracts in the vertical direction. This line is then mapped into a curve which folds producing two disjoint curves $\gamma_{1}^{1}, \gamma_{1}^{2}$ that intersect the original rectangle. Let us denote $s_{1}=F_{0}\left(\gamma_{0}(s)\right)+\varepsilon^{2 / 3} \widetilde{F}\left(s, \gamma_{0}(s)\right)$.

Theorem 5.3. Consider the successive iterates of a chosen initial curve $\gamma_{0}$ intersection the rectangle $\mathbf{R}$ :

$$
\mathbf{R} \cap \mathfrak{S}\left(\gamma_{0}\right)=\gamma_{1}^{1} \cup \gamma_{1}^{2}, \cdots, \mathbf{R} \cap \mathfrak{S}\left(\gamma_{n}^{\sigma_{n}}(s)\right)=\gamma_{n+1}^{\sigma_{n+1}}\left(s_{1}\right) \cup \gamma_{n+1}^{\sigma_{n+1}+1}\left(s_{1}\right),
$$

where $\sigma_{n}=(\underbrace{\cdots, i_{j}, \cdots}_{n}), i_{j}=1,2$ then, for each $\sigma_{n}$ there exists a unique function $t=\gamma_{n}^{\sigma_{n}}(s)$ lying on $\mathbf{R}$ which is invariant under the map $\mathfrak{S}$.

Take an initial curve $t=\gamma_{0}(s)$, its image under $\mathfrak{S}$ noted $\gamma_{1}(s)$ is determined by the functional equation:

$$
G\left(s, \gamma_{0}(s)\right)=\gamma_{1} \circ\left(F\left(s, \gamma_{0}(s)\right)\right.
$$

Lemma 5.4. :

Assume $\gamma_{0}(s)$ is a $\mathbf{C}^{r}(r \geq 3)$ curve with $\left|\gamma_{0}^{\prime}(s)\right| \leq \kappa$ where $\kappa$ is a small positive constant, then $\gamma_{1}^{\prime}(s)$ is also $\mathbf{C}^{r}$ and obeys $\left|\gamma_{1}^{\prime}(s)\right| \leq \kappa$ for $s \in \mathcal{I}_{s}$.

Proof. We will denote $G_{s, t}$ and $F_{s, t}$ the partial derivative with respect to the variables $s, t . \quad M_{\{.\}}$and $N_{\{.\}}$will denote constants. Clearly $\gamma_{1}$ is differentiable since $\left|F_{s}\right|>1$ for $s \in \mathcal{I}_{s}$ and hence $F\left(s, \gamma_{0}(s)\right)$ is invertible in this interval. Let us denote $s_{1}=F\left(\gamma_{0}(s)\right)+\varepsilon^{2 / 3} \widetilde{F}\left(s, \gamma_{0}(s)\right)$. Differentiating (5.9) gives

$$
G_{s}+\gamma_{0}^{\prime}(s) G_{t}=\gamma_{1}^{\prime}\left(s_{1}\right)\left(F_{s}+\gamma_{0}^{\prime}(s) F_{t}\right)
$$

hence

$$
\gamma_{1}^{\prime}\left(s_{1}\right)=\frac{G_{s}+\gamma_{0}^{\prime}(s) G_{t}}{F_{s}+\gamma_{0}^{\prime}(s) F_{t}}
$$

We estimate first the denominator of this expression. For $s \in \mathcal{I}$ we have $\left|F_{s}\right|>$ 1 by proposition (5.1) and by lemma (5.4) $\left|F_{t}\right|<M_{F}$, hence $\left|F_{s}+\gamma_{0}^{\prime}(s) F_{t}\right|>$ $1+\kappa M_{t}>1$. We also know from lemma (5.4) that there exists some constants $M_{G_{s}}<1, M_{G_{t}}<1$ such that $\left|G_{s}\right|<M_{G_{s}}$ and $\left|G_{t}\right|<M_{G_{t}}$, hence the numerator $\left|G_{s}+\gamma_{0}^{\prime}(s) G_{t}\right|<M_{G_{s}}+\kappa M_{G_{t}}<\kappa M_{G_{t}}$. Combining the estimates for the numerator and the denominator we have:

$$
\frac{\left|G_{s}+\gamma_{0}^{\prime}(s) G_{t}\right|}{\left|F_{s}+\gamma_{0}^{\prime}(s) F_{t}\right|}<\kappa M_{G_{t}}<\kappa .
$$

Iterating the same process we can prove $\mathcal{C}^{3}$-regularity.

We can now proceed to the proof of the Theorem. We will denote $|\cdot|_{r}$ the $\mathcal{C}^{r}$ norm. When omitted subscript $|$.$| means the \mathbf{C}^{0}$-norm. Let $\gamma_{0}(s)$ and $\tilde{\gamma}_{0}(s)$ be two curves which have the properties being in $\mathbf{R}$ and obeying $\gamma_{0}^{\prime}(s), \tilde{\gamma}_{0}^{\prime}(s)<\kappa$, then 
their iterates $\gamma_{n}^{\sigma_{n}}(s), \tilde{\gamma}_{n}^{\sigma_{n}}(s)$ will also have these properties by lemma (5.4). We prove now that for $s \in \mathcal{I}_{s}$, the map $\gamma_{n}^{\sigma_{n}}(s) \rightarrow \gamma_{n+1}^{\sigma_{n+1}}\left(s_{1}\right)$ is a contraction in $\mathbf{C}^{3}$.

From the functional equation (5.9) and the fact that $\left|F_{s}\right|>1$ it follows that:

$$
\left|\gamma_{n+1}^{\sigma_{n+1}}\left(s_{1}\right)-\tilde{\gamma}_{n+1}^{\sigma_{n+1}}\left(s_{1}\right)\right| \leq \max _{s \in \mathcal{I}_{s}, t \in \mathcal{I}_{t}}\left|G_{t}\right|\left|\gamma_{n}^{\sigma_{n}}(s)-\tilde{\gamma}_{n}^{\sigma_{n}}(s)\right|
$$

To estimate the derivative we use (5.10) and observe that it is of the form

$$
\gamma_{n+1}^{\sigma_{n+1}}\left(s_{1}\right)=R\left(s, \gamma_{n}^{\sigma_{n}}(s)\right)+\gamma^{\sigma_{n}{ }^{\prime}} S\left(s, \gamma_{n}^{\sigma_{n}}(s)\right)
$$

where the derivatives of $R$ and $S$ are small for $\varepsilon$ small since $\left|F_{s}\right|>1$. Using this form, a mean value estimate gives

$$
\begin{aligned}
(5.13) \gamma_{n+1}^{\sigma_{n+1}{ }^{\prime}}\left(s_{1}\right)-\tilde{\gamma}_{n+1}^{\sigma_{n+1}{ }^{\prime}}\left(s_{1}\right) \mid & \leq c_{1}\left|\gamma_{n}^{\sigma_{n}}(s)-\tilde{\gamma}_{n}^{\sigma_{n}}(s)\right|+c_{2}\left|\gamma_{n}^{\sigma_{n}{ }^{\prime}}(s)-\tilde{\gamma}_{n}^{\sigma_{n}{ }^{\prime}}(s)\right|_{1} \\
& \leq c_{3}\left|\gamma_{n}^{\sigma_{n}}(s)-\tilde{\gamma}_{n}^{\sigma_{n}}(s)\right|_{1},
\end{aligned}
$$

for some constants $c_{1}, c_{2}, c_{3}$.

Using the estimates (5.11) and (5.13) we have that the map $\gamma_{n}^{\sigma_{n}}(s) \rightarrow \gamma_{n+1}^{\sigma_{n+1}}\left(s_{1}\right)$ is a contraction in $\mathcal{C}^{1}$. It then follows in a standard way that the iterates will converge to a unique solution of the functional equation (5.9). It can be seen from (5.12) that higher derivatives are estimated in the same way and we obtain a $\mathcal{C}^{3}$ invariant curve for the 2-D mapping. By construction this curve satisfies:

$$
\mathfrak{S}(s, \gamma(s))=(F(s, \gamma(s)), \gamma \circ F(s, \gamma(s))) \text {. }
$$

This ends the proof of the Theorem (5.3). form

Using this invariant curve $\gamma(s)$, we have that the restricted dynamics is of the

$$
s \mapsto F_{0}(s)+\varepsilon^{2 / 3} \tilde{F}(s, \gamma(s)) .
$$

Since the perturbation is $\mathcal{C}^{3}$ one can compute the Schwartzian derivative which is clearly negative when $\varepsilon$ is sufficiently small. This proves the existence of a chaotic motion in this class of model. Moreover these orbits are confined in a exponentially small region around the orbit of the singular system. This does not preclude the existence of a more complicated invariant chaotic set. In fact the encoding of the intersection of these invariant curves with the $t$-axis, $t=\gamma_{n}^{\sigma_{n}}(s)$ provides a way to describe in detail the foliation of this unstable attractor. A complete study of this set is beyond the scope of this work and is the subject of a forthcoming paper. 


\section{REFERENCES}

[1] Ignacio B. Vivancos, Xiao B. Lin. Heteroclinic and periodic cycles in a perturbed convection model. Journal of Differential Equations, vol. 182, 219-265. (2002)

[2] F.H Busse. K.E Heikes. Convection in a rotating layer: A simple case of turbulence. Science, vol 208. (1980)

[3] P. Chossat, M. Krupa, I. Melbourne, A. Scheel. Transverse bifurcations of homoclinic cycles. Physica D 100, 85-100. (1997)

[4] B. Deng. Spiral-Plus-Saddle attractors and elementary mechanisms for chaos generation. Journal of Bifurcation and Chaos. Vol.6, No.3. pp 513-527. (1996)

[5] J. Guckenheimer. Towards a global model theory of singularly perturbed dynamical systems. Progress in Nonlinear Differential Equations and their applications, vol. 19, 213-225. (1996)

[6] J. Guckenheimer, P. Holmes. Structurally stable heteroclinic cycles. Math. Proc. Camb. Phil. Soc. 103, 189-192. (1988)

[7] M. Krupa, I. Melbourne. Asymptotic Stability of Heteroclinic Cycles in systems with Symmetry. Erg.Th.Dyn.Sys. 15 121-147. (1995)

[8] E.F Mischenko, Yu. S Kolesov, A. Yu Kolesov, N. Kh Rozov. Asymptotic Methods in Singularly Perturbed Systems. Monographs in Contemporary Mathematics. (1993)

[9] B. Sandstede, A. Scheel. Forced symmetry breaking of homoclinic cycles. Nonlinearity 8 , 333-365. (1995)

Departamento de Matemática Aplicada, Facultad de Ciencias Matemáticas, UniverSidad Complutense, 28040 Madrid, Spain.

Departamento de Matemáticas y Mecánica iImAS-UnAm. Apdo. Postal 20-726. México D.F. MÉXICO

E-mail address: Ignacio_Bosch@mat.ucm.es 